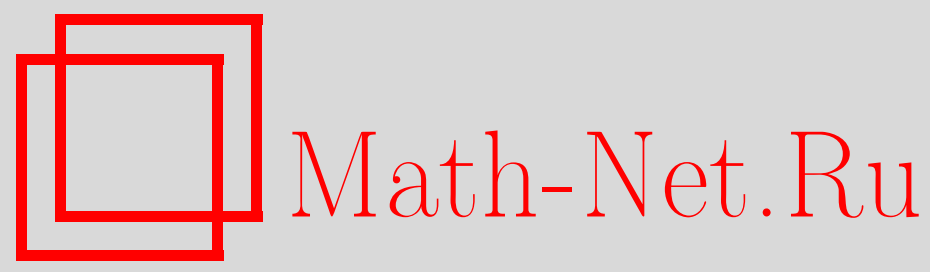

В. Г. Кротов, И. Н. Катковская, Неравенство сильного типа для свертки с корнем квадратным из ядра Пуассона, $\mathrm{Ma-}$ тем. заметки, 2004, том 75, выпуск 4, 580-591

DOI: https://doi.org/10.4213/mzm52

Использование Общероссийского математического портала Math-Net.Ru подразумевает, что вы прочитали и согласны с пользовательским соглашением http://www.mathnet.ru/rus/agreement

Параметры загрузки:

IP : 52.6 .47 .48

26 апреля 2023 г., 10:59:09 


\title{
НЕРАВЕНСТВО СИЛЬНОГО ТИПА ДЛЯ СВЕРТКИ С КОРНЕМ КВАДРАТНЫМ ИЗ ЯДРА ПУАССОНА
}

\author{
И.Н. Катковская, В.Г. Кротов
}

Граничное поведение сверток с ядром Пуассона и с корнем квадратным из ядра Пуассона существенно различно. Первые имеют лишь некасательный предел. Для последних имеет место сходимость по областям, допускающим логарифмический порядок касания с границей (P. Sjögren, J.-O. Rönning). Этот результат был обобщен авторами на пространства однородного типа. Здесь мы доказываем ограниченность в $L^{p}, p>1$, соответствующего максимального оператора. Ранее было известно лишь неравенство слабого типа.

Библиография: 12 названий.

\section{1. Введение}

Пусть

$$
p(z, \theta)=\frac{1}{2 \pi} \cdot \frac{1-|z|^{2}}{\left|z-e^{i \theta}\right|^{2}}
$$

- ядро Пуассона в единичном круге $B$ комплексной плоскости.

Хорошо известно (см., например, [1]), что для любой функции $f \in L^{1}[-\pi, \pi]$ ее интеграл Пуассона

$$
P f(z)=\int_{-\pi}^{\pi} p(z, \theta) f(\theta) d \theta
$$

для почти всех $\varphi \in[-\pi, \pi]$ сходится к $f(\varphi)$, когда $z$ стремится к $e^{i \varphi}$, оставаясь в некасательной области ${ }^{1}$

$$
\left\{z:\left|z-e^{i \varphi}\right|<a\left(1-|z|^{2}\right)\right\}, \quad a>0 .
$$

Литтлвуд [2] (см. также [1]) показал, что это - наилучший результат в следующем смысле. Если $C_{0}-$ любая простая замкнутая кривая, проходящая через точку $z=1$, расположенная целиком, за исключением этой точки, внутри $B$ и касающаяся границы $B$ в этой точке, кривая $C_{\theta}$ получается из $C_{0}$ поворотом вокруг $z=0$ на угол $\theta$, то существует произведение Бляшке, которое для почти всех $\theta$ не стремится ни к какому пределу, если $z \rightarrow e^{i \theta}$, оставаясь внутри $C_{\theta}$.

\footnotetext{
${ }^{1} \mathrm{~B}$ дальнейшем $a$ означает произвольно фиксированную положительную постоянную, а через $c$ (с индексами) мы обозначаем различные положительные постоянные, зависящие, возможно, от некоторых параметров, но эта зависимость для нас несущественна.
} 
В работе [3] было начато изучение граничного поведения сверток со степенями ядра Пуассона

$$
P_{l} f(z)=\int_{-\pi}^{\pi}[p(z, \theta)]^{l+1 / 2} f(\theta) d \theta, \quad l \geqslant 0 .
$$

Интерес к ним объясняется тем, что $P_{l}(z, \cdot)$ (и $\left.P_{l} f(z)\right)$ является решением уравнения

$$
\frac{1}{4}\left(1-|z|^{2}\right)^{2}\left(\frac{\partial^{2} u}{\partial x^{2}}+\frac{\partial^{2} u}{\partial y^{2}}\right)=\left(l^{2}-\frac{1}{4}\right) u
$$

$\left((1 / 4)\left(1-|z|^{2}\right)^{2} \Delta u-\right.$ лапласиан в гиперболической метрике). Конечно, сходимости $P_{l} f(z)$ трудно ожидать без нормировки.

Пусть

$$
\mathscr{P}_{l} f(z)=\frac{P_{l} f(z)}{P_{l} 1(z)} .
$$

Отметим, что ${ }^{2}$

$$
P_{l} 1(z) \asymp \begin{cases}(1-|z|)^{1 / 2-l}, & l>0, \\ (1-|z|)^{1 / 2} \log \frac{2}{1-|z|}, & l=0\end{cases}
$$

(не важно, по какому основанию берутся логарифмы, нам будет удобно всюду считать основание равньп 2).

При $l>0$ граничное поведение интегралов $\mathscr{P}_{l} f(z)$ такое же, как и при $l=1 / 2$ (оно было описано вьше). Иначе обстоит дело при $l=0$. В этом случае граничное поведение операторов (3) изучалось в работах [3]-[7], где было показано, что $\mathscr{P}_{l} f(z)$ сходится к $f(\varphi)$, когда $z$ стремится к $e^{i \varphi}$, оставаясь внутри области

$$
\left\{z \in \mathbb{C}:\left|z-e^{i \varphi}\right|<a(1-|z|)\left(\log \frac{2}{1-|z|}\right)^{p}\right\}
$$

для каждой функции $f \in L^{p}[-\pi, \pi]$ при почти всех $\varphi \in[-\pi, \pi]$. Случай $p=1$ рассмотрен в [3], а $p>1$ - в [5], [6]. Отметим, что области (5) сушественно шире некасательных областей (1) и допускают касательный подход к точке $e^{i \varphi}$, причем большему $p$ соответствует большая степень касания.

Доказательство сходимости почти всюду в работах [3]-[6] опиралось на неравенство слабого типа

$$
\mu\left\{\mathscr{L}_{p}\left(\mathscr{P}_{0} f\right)>\lambda\right\} \leqslant c\left(\frac{1}{\lambda}\|f\|_{L_{\mu}^{p}(X)}\right)^{p}, \quad \lambda>0,
$$

для максимального оператора

$$
\mathscr{L}_{p} f\left(e^{i \varphi}\right)=\sup \left\{|f(z)|:|\arg z-\varphi|<a(1-|z|)\left(\log \frac{2}{1-|z|}\right)^{p}\right\}
$$

соответствующего областям (5). Из (6) результаты о касательной сходимости почти всюду $\mathscr{P}_{l} f(z)$ выводятся стандартным способом. Кроме того, в [5], [6] показано также, что области подхода к границе (5) выбраны оптимально и не могут быть расширены.

\footnotetext{
${ }^{2}$ Запись $f \asymp g$ означает, что существует постоянная $c>0$ такая, что $1 / c \leqslant f / g \leqslant c$.
} 
В нашей работе [8] неравенство (6) было распространено на пространства однородного типа.

Пусть $X$ - компактное хаусдорфово пространство, топология которого задается квазиметрикой $d$. Это означает, что функция $d: X \times X \rightarrow[0, \infty)$ удовлетворяет условиям

$$
d(x, y)=0 \Longleftrightarrow x=y, \quad d(x, y)=d(y, x), \quad d(x, y) \leqslant a_{d}[d(x, z)+d(z, y)]
$$

для любых $x, y, z \in X$ (постоянная $a_{d} \geqslant 1$ не зависит от выбора элементов $x, y, z$ в $X$ ) и семейство открытых шаров

$$
B(x, t)=\{y \in X: d(x, y)<t\}
$$

образует базу окрестностей топологии $X$. В дальнейшем для простоты будем считать, что $\operatorname{diam} X \leqslant 1$.

Пусть еще $\mu$ - положительная борелевская мера на $X$, удовлетворяющая условию однородности

$$
\mu(B(x, t)) \asymp t^{\gamma}
$$

порядка $\gamma>0$ (постоянные слабой эквивалентности в (8) не зависят от $x \in X$ и $t \in$ $(0, \operatorname{diam} X])$. Обычно тройка $(X, d, \mu)$ называется пространством однородного ти$n a$ [9]. Через $L_{\mu}^{p}(X), 1 \leqslant p<\infty$, обозначаем обычные лебеговы пространства, построенные по мере $\mu$.

В этой работе мы рассматриваем операторы

$$
\mathscr{P}_{0} f(x, t)=\left(\log \frac{2}{t}\right)^{-1} \int_{X} \frac{f(y)}{(d(x, y)+t)^{\gamma}} d \mu(y) .
$$

В частном случае $X=\{z \in \mathbb{C}:|z|=1\}, d$ - евклидова метрика и $\mu$-мера Лебега (тогда $\gamma=1$ ) эти операторы совпадают, по существу, с (3) при $l=0$ (см. (4) и ниже раздел 3$)$. Для нас эти операторы представляют интерес как предельньй случай $\alpha=0$ операторов типа потенциала на пространствах однородного типа

$$
\int_{X} \frac{f(y)}{(d(x, y)+t)^{\gamma-\alpha}} d \mu(y), \quad 0<\alpha<\gamma
$$

граничное поведение которых рассматривалось в нашей работе [8].

Введем максимальные функции

$$
\mathscr{L}_{\delta} u(x)=\sup \left\{|u(y, t)|: d(x, y)<a t\left(\log \frac{2}{t}\right)^{\delta}\right\}, \quad a>0, \quad x \in X,
$$

зависящие от параметра $\delta \geqslant 0$. При $\delta=0$ будем обозначать $\mathscr{L}_{0}=N$ (это - "некасательная" максимальная функция).

В частности, в [8] неравенство слабого типа (6) было распространено на общий случай пространств однородного типа

$$
\mu\left\{\mathscr{L}_{\delta}\left(\mathscr{P}_{0} f\right)>\lambda\right\} \leqslant c\left(\frac{1}{\lambda}\|f\|_{L_{\mu}^{p}(X)}\right)^{p}, \quad \lambda>0,
$$


где $\delta=p / \gamma$. При этом в [8] использовался метод, отличный от применявшегося в [3]-[6].

Из теоремы 1 в [8] также можно вывести точные результаты о граничном поведении операторов (3) при $l<0$.

\section{2. Основная теорема и ее доказательство}

Главной целью настоящей работы является доказательство того, что для $p>1$ на самом деле справедливо большее - неравенство (6) можно усилить и заменить неравенством сильного типа, причем в общей ситуации. Именно, имеет место следующее утверждение.

Tеорема 1. Ecлu $p>1 u \delta=p / \gamma, m o$

$$
\left\|\mathscr{L}_{\delta}\left(\mathscr{P}_{0} f\right)\right\|_{L_{\mu}^{p}(X)} \leqslant c_{p}\|f\|_{L_{\mu}^{p}(X)},
$$

где постоянная $c_{p}$ не зависит от $f \in L_{\mu}^{p}(X)$.

В дальнейшем для простоты считаем, что в (10) $a=1$. Для доказательства нам понадобится ряд вспомогательных фактов. Начнем с хорошо известных.

Лемма 1. Пусть $E \subset X u\{B\}$ - любое семейство шаров ограниченных радиусов, покрывающее $E$.

Тогда существует конечное или счетное подсемейство $\left\{B_{j}\right\} \subset\{B\}$ со свойствами

$$
B_{i} \cap B_{j}=\varnothing, \quad i \neq j, \quad E \subset \bigcup_{j} \rho_{d} B_{j},
$$

где $\rho_{d} \geqslant 1$ зависит только от $d$.

Здесь $\rho B$ - шар с тем же центром, что и $B$, радиуса в $\rho$ раз больше. Доказательство леммы имеется в [9].

С помощью леммы 1 стандартным способом [9] выводятся обычные свойства максимальной функции Харди-Литтлвуда

$$
M f(x)=\sup \frac{1}{\mu(B)} \int_{B}|f| d \mu,
$$

где точная верхняя грань берется по всем шарам $B=B(y, t)$, содержащим точку $x \in X$.

ЛЕмма 2. Для каждого $p \geqslant 1$ существует такая постоянная $c_{p}$, что

1) для всех функиий $f \in L_{\mu}^{1}(X) u \lambda>0$

$$
\mu\{M f>\lambda\} \leqslant \frac{c_{1}}{\lambda}\|f\|_{L_{\mu}^{1}(X)},
$$

2) для всех функиий $f \in L_{\mu}^{p}(X)$

$$
\|M f\|_{L_{\mu}^{p}(X)} \leqslant c_{p}\|f\|_{L_{\mu}^{p}(X)} .
$$


Введем параметрическое семейство областей подхода к гранище

$$
D_{A, \delta}(x)=\left\{(y, t): d(x, y)<t\left(\log \frac{2}{t}\right)^{\delta}, A<\left(\log \frac{2}{t}\right)^{\delta}\right\}
$$

и отметим, что второе неравенство, определяющее $D_{A, \delta}(x)$, равносильно следующему неравенству:

$$
t<\exp \left(1-A^{1 / \delta}\right)=\tau_{A} .
$$

Определим также семейство максимальных функций

$$
\mathscr{L}_{A, \delta} u(x)=\sup \left\{\left(\log \frac{2}{t}\right)^{-1} u(y, A t):(y, t) \in D_{A, \delta}(x)\right\},
$$

с помощью которых можно оценить оператор $\mathscr{L}_{\delta}\left(\mathscr{P}_{0} f\right)$. Это показывает лемма 3 ниже, в которой используется следующее обозначение:

$$
u(y, t)=\frac{1}{\mu(B(y, t))} \int_{B(y, t)}|f| d \mu,
$$

где $f \in L_{\mu}^{1}(X)$. Тогда ясно, что

$$
N u(x)=M f(x), \quad x \in X .
$$

Лемма 3. Существует такая постоянная с, что

$$
\mathscr{L}_{\delta}\left(\mathscr{P}_{0} f\right)(x) \leqslant c\left(M f(x)+\sum_{\nu=0}^{\infty} \mathscr{L}_{2^{\nu}, \delta} u(x)\right)
$$

для всех $x \in X u f \in L_{\mu}^{1}(X)$.

ДокАЗАТЕЛЬСтво. Пусть $x \in X$ и пара $(y, t) \in X \times(0,1)$ удовлетворяет условию

$$
d(x, y)<\tau=t\left(\log \frac{2}{t}\right)^{\delta}
$$

Разобьем интеграл, определяющий оператор (9), на три части

$$
\int_{X} \frac{f(z)}{(d(y, z)+t)^{\gamma}} d \mu(z)=\int_{B(y, t)}+\int_{t<d(x, y) \leqslant \tau}+\int_{d(x, y)>\tau} \equiv I_{1}+I_{2}+I_{3}
$$

и каждую из них будем оценивать отдельно.

Прежде всего заметим, что

$$
\left|I_{1}\right| \leqslant u(y, t) .
$$

Кроме того, если $n=\left[\log _{2}(\tau / t)\right]+2$, то

$$
\begin{aligned}
\left|I_{2}\right| & \leqslant \sum_{\nu=0}^{n-1} \int_{2^{\nu} t<d(y, z) \leqslant 2^{\nu+1} t} \frac{f(z)}{(d(y, z)+t)^{\gamma}} d \mu(z) \leqslant \sum_{\nu=0}^{n-1}\left(2^{\nu} t\right)^{\gamma} \int_{B\left(y, 2^{\nu+1} t\right)}|f| d \mu \\
& \leqslant c \sum_{\nu=0}^{n-1} \frac{1}{\mu B\left(y, 2^{\nu+1} t\right)} \int_{B\left(y, 2^{\nu+1} t\right)}|f| d \mu \leqslant c \sum_{\nu=1}^{n} u\left(y, 2^{\nu} t\right) .
\end{aligned}
$$


Наконец, если $m=\left[\log _{2}(1 / \tau)\right]+2$, то

$$
\begin{aligned}
\left|I_{3}\right| & \leqslant \sum_{\nu=0}^{m-1} \int_{2^{\nu} \tau<d(y, z) \leqslant 2^{\nu+1} \tau} \frac{f(z)}{(d(y, z)+t)^{\gamma}} d \mu(z) \leqslant \sum_{\nu=0}^{m-1}\left(2^{\nu} \tau\right)^{\gamma} \int_{B\left(y, 2^{\nu+1} \tau\right)}|f| d \mu \\
& \leqslant c \sum_{\nu=0}^{m-1} \frac{1}{\mu B\left(y, 2^{\nu+1} \tau\right)} \int_{B\left(y, 2^{\nu+1} \tau\right)}|f| d \mu \leqslant c m M f(x) \leqslant c \log \frac{1}{\tau} M f(x) .
\end{aligned}
$$

Из полученных неравенств для $I_{1}, I_{2}$ и $I_{3}$ легко вытекает утверждение леммы 3 .

Следующая лемма играет ключевую роль. При ее доказательстве мы будем следовать схеме рассуждений из работы второго автора [10], видоизменяя ее соответственно рассматриваемой ситуации.

ЛЕмма 4 (основная). Для каждого $p>1$ существует такая постоянная $c_{p}$, чmo

$$
\left\|\mathscr{L}_{A, \delta} u\right\|_{L_{\mu}^{p}(X)} \leqslant c_{p} A^{-\gamma / p}\|f\|_{L_{\mu}^{p}(X)}
$$

при всех $f \in L_{\mu}^{p}(X)$ и $A \geqslant 1$.

ДокАЗАтЕльСтво. Рассмотрим лебеговы множества максимального оператора (14)

$$
E_{A}(\lambda)=\left\{x \in X: \mathscr{L}_{A, \delta} u(x)>\lambda\right\}, \quad \lambda>0 .
$$

Разобьем их на части следуюшим образом. Определим натуральное число $k_{A}$ из условия $2^{-k_{A}-1}<\tau_{A} \leqslant 2^{-k_{A}}$, обозначим

$$
t_{A}(x)=\sup \left\{t<\tau_{A}: \exists(y, t) \in D_{A, \delta}(x), \quad\left(\log \frac{2}{t}\right)^{-1} u(y, A t)>\lambda\right\}
$$

и для $k \geqslant k_{A}$ введем множества

$$
E_{A, k}(\lambda)=\left\{x \in E_{A}(\lambda): t_{A}(x) \in\left(2^{-k-1}, 2^{-k}\right]\right\} .
$$

Они измеримы и

$$
E_{A, k}(\lambda) \cap E_{A, i}(\lambda)=\varnothing, \quad E_{A}(\lambda)=\bigcup_{k=k_{A}}^{\infty} E_{A, k}(\lambda) .
$$

Введем также некоторую модификацию некасательной максимальной функции

$$
N_{A} u(x)=\sup \left\{u(y, A \tau): d(x, y)<\frac{A \tau}{4 a_{d}^{2}}, \quad \tau<\tau_{A}\right\} .
$$

Тогда ясно, что

$$
N_{A}(x) \leqslant N(x), \quad x \in X .
$$

Как и вьше, введем лебеговы множества

$$
E(\lambda)=\left\{x \in X: N_{A} u(x)>\lambda\right\}
$$


и разобьем их на части

$$
E_{k}(\lambda)=\left\{x \in E(\lambda): \tau(x) \in\left(2^{-k-1}, 2^{-k}\right]\right\}
$$

где

$$
\tau(x)=\sup \left\{\tau<\tau_{A}: \exists y \quad d(x, y)<\frac{A \tau}{4 a_{d}^{2}}, \quad u(y, A \tau)>\lambda\right\}
$$

Тогда множества $E_{k}(\lambda)$ измеримы и

$$
E_{k}(\lambda) \cap E_{i}(\lambda)=\varnothing, \quad E(\lambda)=\bigcup_{k=k_{A}}^{\infty} E_{k}(\lambda)
$$

Оценим меру $\mu E_{A, k}(\lambda)$. Пусть $x \in E_{A, k}(\lambda)$; тогда существует такая пара $\left(y_{x}, t_{x}\right) \in$ $X \times\left(2^{-k-1}, 2^{-k}\right]$, что

$$
d\left(x, y_{x}\right)<t_{x}\left(\log \frac{2}{t_{x}}\right)^{\delta}, \quad u\left(y_{x}, A t_{x}\right)>\lambda \log \frac{2}{t_{x}} \geqslant k \lambda .
$$

Рассмотрим семейство шаров

$$
B_{x}=B\left(y_{x}, t_{x}\left(\log \frac{2}{t_{x}}\right)^{\delta}\right), \quad x \in E_{A, k}(\lambda)
$$

По лемме 1 из него можно выделить конечное или счетное подсемейство $\left\{B_{x_{j}}\right\}$ со свойствами

$$
B_{x_{j}} \cap B_{x_{i}}=\varnothing, \quad \mu E_{A, k}(\lambda) \leqslant c \sum_{j} \mu B_{x_{j}} .
$$

Введем новое семейство шаров

$$
B_{x_{j}}^{*}=B\left(y_{x_{j}}, A t_{x_{j}}\right), \quad j \geqslant 1
$$

Пусть

$$
\varphi(t)=t\left(\log \frac{2}{t}\right)^{\delta}
$$

тогда, как легко видеть, существует такое $k_{0} \in \mathbb{N}$, что

$$
\varphi(t)<\frac{\varphi(\tau)}{4 a_{d}^{2}} \quad \text { при } \quad 2^{k_{0}} t<\tau
$$

(cм. (7)).

Покажем, что справедливы включения

$$
B_{x_{j}}^{*} \subset \bigcup_{i=k-k_{0}}^{k} E_{i}(k \lambda), \quad k \geqslant k_{0}+k_{A} .
$$


Пусть $x \in B_{x_{j}}^{*}$ и пара $(z, \tau)$ такова, что

$$
d(x, z)<\frac{A \tau}{2 a_{d}^{2}}, \quad 2^{k_{0}-k}<\tau<\tau_{A}
$$

Тогда в силу $(7),(13),(18)$ и (19)

$$
\begin{aligned}
d\left(x_{j}, z\right) & \leqslant a_{d}^{2}\left[d\left(x_{j}, y_{j}\right)+d\left(y_{j}, x\right)+d(x, z)\right] \\
& \leqslant a_{d}^{2}\left[t_{x_{j}}\left(\log \frac{2}{t_{x_{j}}}\right)^{\delta}+A t_{x_{j}}+\frac{A \tau}{2 a_{d}^{2}}\right]<\tau\left(\log \frac{2}{\tau}\right)^{\delta} .
\end{aligned}
$$

Следовательно, $(z, \tau) \in D_{A, \delta}\left(x_{j}\right)$ (см. (12)), но $x_{j} \in E_{A, k}(\lambda)$ и $2^{k_{0}-k}<\tau<\tau_{A}$, поэтому

$$
u(z, A \tau) \leqslant \lambda \log \frac{2}{\tau}<\lambda \log 2^{k-k_{0}+1}<k \lambda .
$$

Это означает, что $x \notin E_{i}(k \lambda)$ при $i<k-k_{0}$.

$\mathrm{C}$ другой стороны, так как $t_{x_{j}}>2^{-k-1}, d\left(x, y_{j}\right)<A t_{x_{j}}$, то из второго неравенства (18) вытекает, что $x \notin E_{i}(k \lambda)$ при $i>k$. Таким образом, включение (20) доказано.

Теперь, используя (20), получаем при $k \geqslant k_{0}+k_{A}$

$$
\begin{aligned}
\mu E_{A, k}(\lambda) & \leqslant c \sum_{j} \mu B_{x_{j}}=c \sum_{j} \frac{\mu B_{x_{j}}}{\mu B_{x_{j}}^{*}} \cdot \mu B_{x_{j}}^{*}=c k^{p} A^{-\gamma} \sum_{j} \mu B_{x_{j}}^{*} \\
& =c k^{p} A^{-\gamma} \mu\left(\bigcup_{j} B_{x_{j}}^{*}\right) \leqslant c k^{p} A^{-\gamma} \mu\left(\bigcup_{i=k-k_{0}}^{k} E_{i}(k \lambda)\right) .
\end{aligned}
$$

Подобным образом, но с существенньми упрощениями, доказьвается и неравенство

$$
\mu\left(\bigcup_{k=k_{A}}^{k_{A}+k_{0}-1} E_{A, k}(\lambda)\right) \leqslant c k_{A}^{p} A^{-\gamma} \mu\left(\bigcup_{i=k_{A}}^{k_{A}+k_{0}-1} E_{i}(k \lambda)\right) .
$$

В самом деле, пусть для краткости

$$
S_{A}(\lambda)=\bigcup_{k=k_{A}}^{k_{A}+k_{0}-1} E_{A, k}(\lambda)
$$

и $x \in S_{A}(\lambda)$, тогда существует такая пара $\left(y_{x}, t_{x}\right) \in X \times\left(2^{-k_{A}-k_{0}}, \tau_{A}\right]$, что

$$
d\left(x, y_{x}\right)<t_{x}\left(\log \frac{2}{t_{x}}\right)^{\delta}, \quad u\left(y_{x}, A t_{x}\right)>\lambda \log \frac{2}{t_{x}} \geqslant k \lambda .
$$

Как и вьше, рассмотрим семейство шаров

$$
B_{x}=B\left(y_{x}, t_{x}\left(\log \frac{2}{t_{x}}\right)^{\delta}\right), \quad x \in E_{A, k}(\lambda)
$$


По лемме 1 из него можно выделить конечное или счетное подсемейство $\left\{B_{x_{j}}\right\}$ со свойствами

$$
B_{x_{j}} \cap B_{x_{i}}=\varnothing, \quad \mu S_{A}(\lambda) \leqslant c \sum_{j} \mu B_{x_{j}} .
$$

Введем новое семейство шаров

$$
B_{x_{j}}^{*}=B\left(y_{x_{j}}, A t_{x_{j}}\right), \quad j \geqslant 1
$$

тогда в силу (23)

$$
B_{x_{j}}^{*} \subset \bigcup_{k=k_{A}}^{k_{A}+k_{0}-1} E_{k}(\lambda) .
$$

Повторяя доказательство неравенства (21), получим (22).

Из (21) и (22) нетрудно вывести оценку для нормы

$$
\begin{aligned}
\left\|\mathscr{L}_{A, \delta} u\right\|_{L_{\mu}^{p}(X)}^{p} & =p \int_{0}^{\infty} \lambda^{p-1} \mu E_{A}(\lambda) d \lambda=p \sum_{k=k_{A}}^{\infty} \int_{0}^{\infty} \lambda^{p-1} \mu E_{A, k}(\lambda) d \lambda \\
& \leqslant c \sum_{k=k_{A}}^{\infty} k^{p} A^{-\gamma} \sum_{i=k-k_{0}}^{k} \int_{0}^{\infty} \lambda^{p-1} \mu E_{i}(k \lambda) d \lambda .
\end{aligned}
$$

Заменяя в последнем интеграле $k \lambda$ на новое $\lambda$, получим (см. еще $(17),(15)$ и лемму 2$)$

$$
\begin{aligned}
\left\|\mathscr{L}_{A, \delta} u\right\|_{L_{\mu}^{p}(X)}^{p} & \leqslant c A^{-\gamma} \sum_{k=k_{A}}^{\infty} \int_{0}^{\infty} \lambda^{p-1} \sum_{i=k-k_{0}}^{k} \mu E_{i}(\lambda) d \lambda \\
& \leqslant c A^{-\gamma} \int_{0}^{\infty} \lambda^{p-1} \sum_{i=0}^{\infty} \mu E_{i}(\lambda) d \lambda \\
& =c A^{-\gamma} \int_{0}^{\infty} \lambda^{p-1} \mu E(\lambda) d \lambda=c A^{-\gamma}\left\|N_{A} u\right\|_{L_{\mu}^{p}(X)}^{p}=c A^{-\gamma}\|M f\|_{L_{\mu}^{p}(X)}^{p} \\
& \leqslant c A^{-\gamma}\|f\|_{L_{\mu}^{p}(X)}^{p},
\end{aligned}
$$

и лемма 4 доказана.

Утверждение теоремы 1 вытекает теперь непосредственно из лемм 2-4.

\section{3. Некоторые обобщения и частные случаи}

3.1. Локальная форма теоремы 1. Прежде всего отметим, что доказательство теоремы 1 носит локальньй характер. Это позволяет доказать следующее обобщение теоремы 1.

ТЕОрема 2. Пусть $G \subset X$ - открытое множсество и $p>1$. Тогда для любого компакта $K \subset G$ существует такая постоянная $c_{p}(K)$, что для $f \in L_{\mu}^{1}(X) \cap L_{\mu}^{p}(G)$ выполнено неравенство

$$
\left\|\mathscr{L}_{\delta}\left(\mathscr{P}_{0} f\right)\right\|_{L_{\mu}^{p}(K)} \leqslant c_{p}(K)\left(\|f\|_{L_{\mu}^{1}(X)}+\|f\|_{L_{\mu}^{p}(G)}\right)
$$


ДоКАЗАТЕЛЬСТВО в значительной степени повторяет рассуждения, проведенные выше. Поэтому мы остановимся кратко лишь на изменениях, которые надо сделать.

Пусть число $\varepsilon>0$ достаточно мало. Разобьем оператор (9) на две части

$$
\mathscr{P}_{0} f(x, t)=\int_{B(x, \varepsilon)} \frac{f(y)}{(d(x, y)+t)^{\gamma}} d \mu(y)+\int_{X \backslash B(x, \varepsilon)} \frac{f(y)}{(d(x, y)+t)^{\gamma}} d \mu(y)
$$

Второе слагаемое оценивается сверху через $c\|f\|_{L_{\mu}^{1}(X)}$ равномерно по $x$ и $t$, а первое оценивается точно так же, как и выше (см. леммы 3 и 4), только вместо максимальной функции Харди-Литтлвуда $M f$ будет участвовать усеченная максимальная функция

$$
M_{\varepsilon} f(x)=\sup \frac{1}{\mu(B)} \int_{B}|f| d \mu
$$

где точная верхняя грань берется по всем шарам $B=B(y, t)$ радиуса $0<t<\varepsilon$, содержащим точку $x \in X$.

3.2. Весовая форма теоремы 1. Используя то же доказательство, можно получить весовой вариант теоремы 1. Для формулировки нам понадобятся некоторые определения.

Неотрищательная функция $\nu$, определенная на борелевских множествах из $X$, называется внешней мерой, если она монотонна и субадлитивна, т.е.

$$
G_{1} \subset G_{2} \Rightarrow \nu\left(G_{1}\right) \leqslant \nu\left(G_{2}\right), \quad \nu\left(\bigcup_{j} G_{j}\right) \leqslant \sum_{j} \nu\left(G_{j}\right)
$$

Если $f$ - борелевская функция и $\nu$ - внешняя мера на $X$, то положим

$$
\|f\|_{L_{\nu}^{p}(X)}=\left(p \int_{0}^{\infty} \lambda^{p-1} \nu\{|f|>\lambda\} d \lambda\right)^{1 / p}
$$

Для меры $\nu$ это обычная норма в $L_{\nu}^{p}(X)$.

ТЕОрема 3. Пусть $p>1,0 \leqslant \delta \leqslant p / \gamma, \beta=p-\gamma \delta u \nu-$ внешняя мера, удовлетворяющая условию

$$
\nu(B(x, t)) \leqslant c t^{\gamma}\left(\log \frac{2}{t}\right)^{\beta}
$$

(с не зависит от $x \in X u t>0$ ).

Тогда

$$
\left\|\mathscr{L}_{\delta}\left(\mathscr{P}_{0} f\right)\right\|_{L_{\nu}^{p}(X)} \leqslant c_{p}\|f\|_{L_{\mu}^{p}(X)}
$$

əде постоянная $c_{p}$ не зависит от $f \in L_{\mu}^{p}(X)$. 
ДоКАЗАТЕЛЬСТво слово в слово копирует обоснование теоремы 1 , лишь в момент оценки отношения мер $\nu B_{x_{j}}$ и $\mu B_{x_{j}}^{*}$ в $(21)$ и (22) надо дополнительно использовать $(24)$. Тогда мы получим

$$
\begin{aligned}
\frac{\nu B_{x_{j}}}{\mu B_{x_{j}}^{*}} & \leqslant \frac{1}{\left(A t_{j}\right)^{\gamma}} \cdot c t_{j}^{\gamma}\left(\log \frac{2}{t_{j}}\right)^{\gamma \delta}\left(\log \frac{2}{t_{j}\left(\log \left(2 / t_{j}\right)\right)^{\delta}}\right)^{\beta} \leqslant \frac{c\left(\log \left(2 / t_{j}\right)\right)^{\gamma \delta+\beta}}{A^{\gamma}} \\
& \leqslant c k^{\gamma \delta+\beta} A^{-\gamma}=c k^{p} A^{-\gamma}
\end{aligned}
$$

Оставшаяся часть доказательства проходит без изменения.

Теорема 1 является частным случаем при $\beta=0$ теоремы 3. Последней также можно придать локальньй характер в духе теоремы 2.

3.3. Многомерные аналоги оператора (3). Рассмотрим, наконец, два частных случая оператора (9), каждый из которых обобщает оператор (3) с $\lambda=0$ на многомерный случай. $\mathrm{K}$ ним применимы теоремы $1-3$.

Пусть $X=S^{n-1}$ - единичная сфера в $\mathbb{R}^{n}, n \geqslant 2, \mu$-поверхностная мера Лебега на $S^{n-1}$, нормированная условием $\mu\left(S^{n-1}\right)=1, d(x, y)=|x-y|$ - евклидова метрика. Тогда в условии (8) $\gamma=n-1$.

Многомерным аналогом (2) будет оператор

$$
P_{l} f(x)=\int_{S^{n-1}}[p(x, \theta)]^{l+(n-1) / n} f(\theta) d \mu(\theta),
$$

где

$$
p(x, \theta)=\frac{1-|x|^{2}}{|x-\theta|^{n}}
$$

- ядро Пуассона для единичного шара (см., например, [11]). При выбранных обозначениях (9) имеет вид

$$
\begin{aligned}
\mathscr{P}_{0} f(x) & =\left(\log \frac{2}{1-|x|}\right)^{-1} \int_{S^{n-1}} \frac{f(\eta)}{|x-\eta|^{n-1}} d \mu(\eta) \\
& \asymp\left(\log \frac{2}{t}\right)^{-1} \int_{S^{n-1}} \frac{f(\eta)}{(|\theta-\eta|+t)^{n-1}} d \mu(\eta),
\end{aligned}
$$

где $t=1-|x|, \theta=x /|x|$.

Пусть $X=S^{2 n-1}-$ единичная сфера в $\mathbb{C}^{n}=\mathbb{R}^{2 n}, \mu$ - поверхностная мера Лебега, $\mu\left(S^{n-1}\right)=1, d(\zeta, \xi)=|1-\langle\zeta, \xi\rangle|-$ неизотропная квазиметрика (здесь $\langle\cdot, \cdot\rangle-$ комплексное скалярное произведение). В этом случае $\gamma=n$.

Сейчас естественным является также инвариантное ядро Пуассона [12]

$$
P_{n}(z, \zeta)=\frac{\left(1-|z|^{2}\right)^{n}}{|1-\langle z, \zeta\rangle|^{2 n}}
$$

и по аналогии с (2) мы приходим к

$$
P_{l} f(z)=\int_{S^{2 n-1}}[p(z, \eta)]^{l+1 / 2} f(\eta) d \mu(\eta) .
$$


Оператор (9) принимает вид

$$
\begin{aligned}
\mathscr{P}_{0} f(z) & =\left(\log \frac{2}{1-|z|}\right)^{-1} \int_{S^{2 n-1}} \frac{f(\eta)}{|1-\langle z, \eta\rangle|^{n}} d \mu(\eta) \\
& \asymp\left(\log \frac{2}{t}\right)^{-1} \int_{S^{2 n-1}} \frac{f(\eta)}{(|1-\langle\zeta, \eta\rangle|+t)^{n}} d \mu(\eta)
\end{aligned}
$$

где $t=1-|z|, \zeta=z /|z|$.

В заключение отметим, что возможны и другие приложения теорем $1-3$. Примерами могут быть граничное поведение интегралов Пуассона в поликруге или на симметрических римановых пространствах (см. [5]-[7]).

\section{СПИСОК ЦИТИРОВАННОЙ ЛИТЕРАТУРЫ}

[1] Зигмунд А. Тригонометрические ряды. Т. 1. М.: Мир, 1965.

[2] Littlewood J. E. On a theorem of Fatou // J. London Math. Soc. 1927. V. 2. P. 172-176.

[3] Sjögren P. Une remarque sur la convergence des fonctions propres du Laplasian à valeur propre critique // Lecture Notes in Math. V. 1096. Berlin: Springer, 1984. P. 544-548.

[4] Sjögren P. Convergence results for the square root of the Poisson kernel // Pacific J. Math. 1988. V. 131. P. 361-391.

[5] Rönning J.-O. Convergence results for the square root of the Poisson kernel // Ph.D. Thesis. Göteborg: Chalmers Univ. Tehnology, 1993.

[6] Rönning J.-O. Convergence results for the square root of the Poisson kernel // Math. Scand. 1997. V. 81. P. 219-235.

[7] Sjögren P. Approach regions for the square root of the Poisson kernel and bounded functions // Bull. Austral. Math. Soc. 1997. V. 55. P. 521-527.

[8] Катковская И. Н., Кротов В. Г. О касательном граничном поведении потенциалов // Тр. ИМ НАН Беларуси. 2000. Т. 5. С. 80-83.

[9] Coifman R.R., Weiss G. Extensions of Hardy spaces and their use in analysis // Bull. Amer. Math. Soc. 1977. V. 83. № 4. P. 569-645.

[10] Кротов В. Г. Оценки для максимальных операторов, связанных с граничным поведением, и их приложения // Тр. МИАН. 1989. Т. 190. С. 117-138.

[11] Стейн И., Вейс Г. Введение в гармонический анализ на евклидовых пространствах. М.: Мир, 1974.

[12] Рудин У. Теория функций в единичном шаре в $\mathbb{C}^{n}$. М.: Мир, 1984.

(И.Н. Катковская) Белорусский государственный

Поступило технический университет, г. Минск 23.06.2003

(В.Г. Кротов) Белорусский государственный университет, г. Минск

E-mail: krotov@bsu.by 